\title{
MINIATURE MODULES FOR MULTI-LEAD ECG RECORDING
}

\author{
Yi-Li Tseng*, Hung-Wei Chiu ${ }^{\dagger}$, Tsung-Hsien Lin ${ }^{\ddagger}$ and Fu-Shan Jaw ${ }^{*}, \S$ \\ *Institute of Biomedical Engineering, \\ National Taiwan University, Taipei, Taiwan \\ ${ }^{\dagger}$ Graduate Institute of Computer and Communication Engineering, \\ National Taipei, University of Technology, Taipei, Taiwan \\ ${ }^{\ddagger}$ Department of Electrical Engineering, \\ National Taiwan University, Taipei, Taiwan \\ §jaw@ntu.edu.tw
}

Accepted 26 May 2008

\begin{abstract}
Remote monitoring systems for home health care service have become one of the hottest topics recently. Biomedical signals recorded by portable devices can be wirelessly transmitted through the Internet. In this paper, a miniature signal-condition module for ambulatory recording of electrocardiogram (ECG) signals was designed with high input impedance, high common-mode rejection ratio (CMRR), low power, appropriate amplification and filtration, and automatic suppression of offset voltage. For early detection of acute myocardial infarction (AMI), this device is extended and 12-lead ECG recording is available. Due to the modular approach, the module is accommodated for other biomedical signals recording as well if the gain and pass-band of the module are modified.
\end{abstract}

Keywords: Signal condition; ECG; Portable; Amplifier.

\section{INTRODUCTION}

Utilizing biomedical signals for detecting and diagnosing diseases is a growing trend today. Recently, ambulatory recorders are used in the application of home health care service. ${ }^{1-3}$ Such a recording system is beneficial to provide early detection of acute diseases. To meet the requirement of remote monitoring, these devices are usually compact ${ }^{4,5}$ and have low-power consumption. ${ }^{6-9}$ In addition, they should provide appropriate filtration and amplification before A/D conversion and wireless transmission. ${ }^{2}$ Circuitry with high common-mode rejection ratio (CMRR) and a suitable pass-band should be also important to prevent the signals from noise interference and frequency distortion. ${ }^{10}$ Further, electrical isolation should be provided to protect patients from electrical shocks.

This investigation focuses on developing an ambulatory recorder for the early detection of acute myocardial infarction (AMI). The 12-lead electrocardiogram (ECG) is the cornerstone for the early diagnosis of AMI. ${ }^{11,12}$ In this paper, a one-channel module for the recording of ambulatory ECG is proposed. A portable device with three modules can measure 12-lead ECG in real-time for monitoring AMI or other heart diseases. This module consists a high input impedance instrumentation amplifier (IA) with auto-DC suppression, ${ }^{13}$ as well as stages of voltage gain and filters. For size and weight reduction, surface-mounted devices (SMDs)

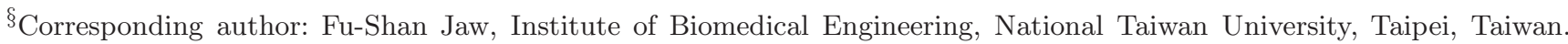
Tel: +886-2-23687401; Fax: +886-2-33665268; E-mail: jaw@ntu.edu.tw. 
were used in the implementation. After optimal tuning and fully debugging, the device was flexible and reliable. Furthermore, after modifications, this module was also involved in the recording of electromyogram (EMG) and electrogastrogram (EGG) ${ }^{4,5}$ The properties of small size and low power are the advantages for portable applications, that fulfill the goal of remote monitoring.

\section{CIRCUIT DESIGN}

The complete circuit of this module is illustrated in Fig. 1. Considering the amplitude of ECG is often between 80 and $2000 \mu \mathrm{V}$ with a bandwidth in the range of $0.01-150 \mathrm{~Hz},{ }^{14}$ the pre-amplifier should be featured with low noise, high CMRR, and low output impedance. A micro-power IA, AD627B, was utilized to fulfill these requirements. The minimal $\mathrm{CMRR}$ of $\mathrm{AD} 627 \mathrm{~B}$ is up to $83 \mathrm{~dB}$ with a good DC accuracy. Further, the typical static current of the AD627B is less than $85 \mu \mathrm{A}$ and consequently suitable for portable applications. The gain of AD627B can be set with an external resistor only and is programmed at 65 here. An integrator was designed that formed a negative-feedback loop to cancel the offset voltage of the IA automatically. ${ }^{13}$ Hence, the circuitry of the first stage provides a gain of 65 , a high-pass filtration with corner frequency of $0.01 \mathrm{~Hz}$, and most important of all, an excellent high input impedance.

The second stage is a noninverting amplifier implemented by an operational amplifier, 1/2 OP296, with a low static current of $60 \mu \mathrm{A}$ only. A fixed gain of 6.1 was designed in this stage to make an overall gain of 400(65 $\times 6.1$ ). The last stage is a second order Butterworth low-pass filter, for its flatness in frequency response.

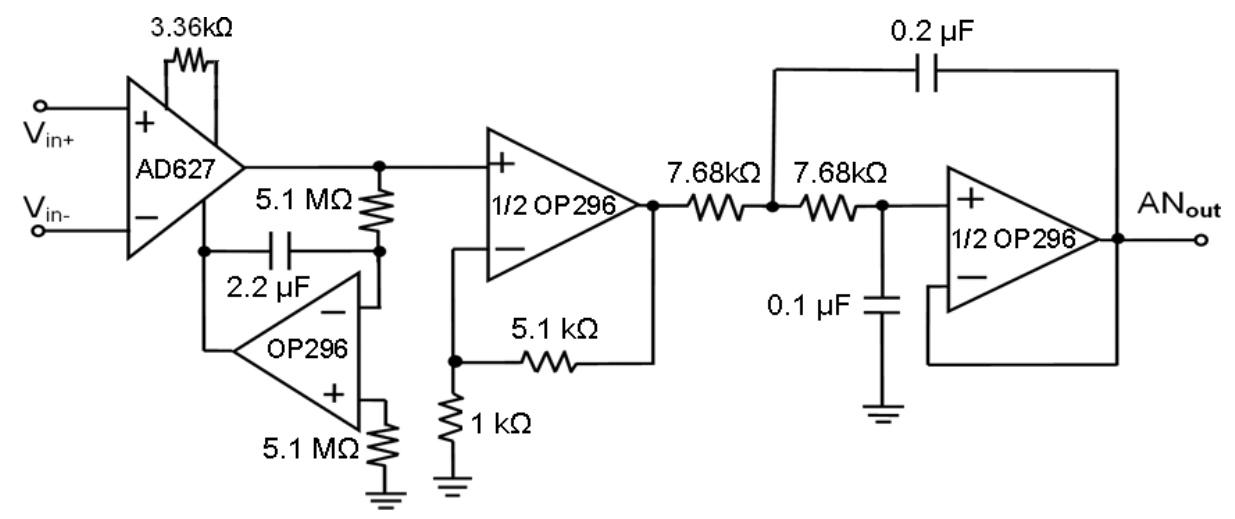

Fig. 1 Circuitry of the module. The 1st stage is the preamplifier consisting of an IA and DC suppression. The 2nd stage is a non-inverting amplifier and the last stage was a second-order low-pass filter.

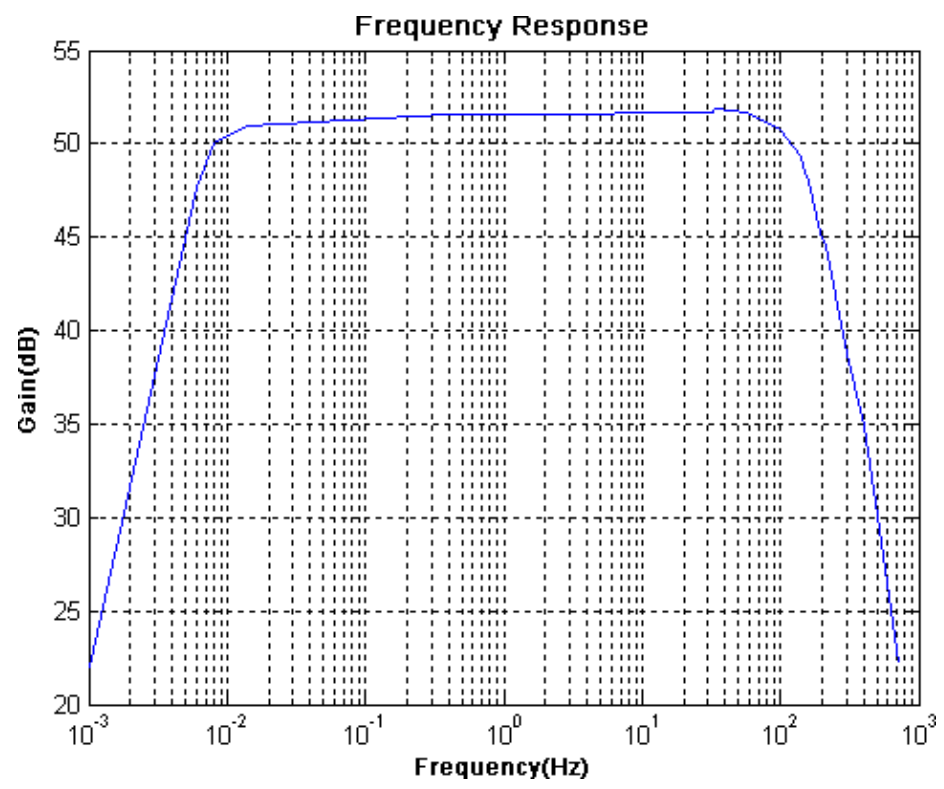

Fig. 2 Frequency response of the module. The actual bandwidth is $0.01-146 \mathrm{~Hz}$ and the gain is $51.6 \mathrm{~dB}$. 
The filter also serves as an anti-aliasing filter to avoid aliasing errors caused by the sampling process of $\mathrm{A} / \mathrm{D}$ conversion. Since the device is powered by batteries, it is electrically isolated in nature and can protect the patient from macro-shock as well. ${ }^{10}$

\section{RESULTS}

The dimension of the signal-condition unit is $36 \mathrm{~mm}$ $\times 15 \mathrm{~mm} \times 2 \mathrm{~mm}$. Figure 2 illustrates the frequency response of the device with a bandwidth of $0.01-146 \mathrm{~Hz}$ and an overall gain of 378 . The quiescent current of a module is only $244 \mu \mathrm{A}$. The circuit is powered by two AAA batteries. The output signal of this module can be either transmitted to the computer wirelessly or directly displayed on the screen of an oscilloscope. A preliminary trial for practical measurement is shown in Fig. 3. A normal lead-II ECG displayed on an oscilloscope is shown in Fig. 3(A), and three-lead ECG (lead I, lead II, and lead III) transmitted via wireless transmission is shown in Fig. 3(B).

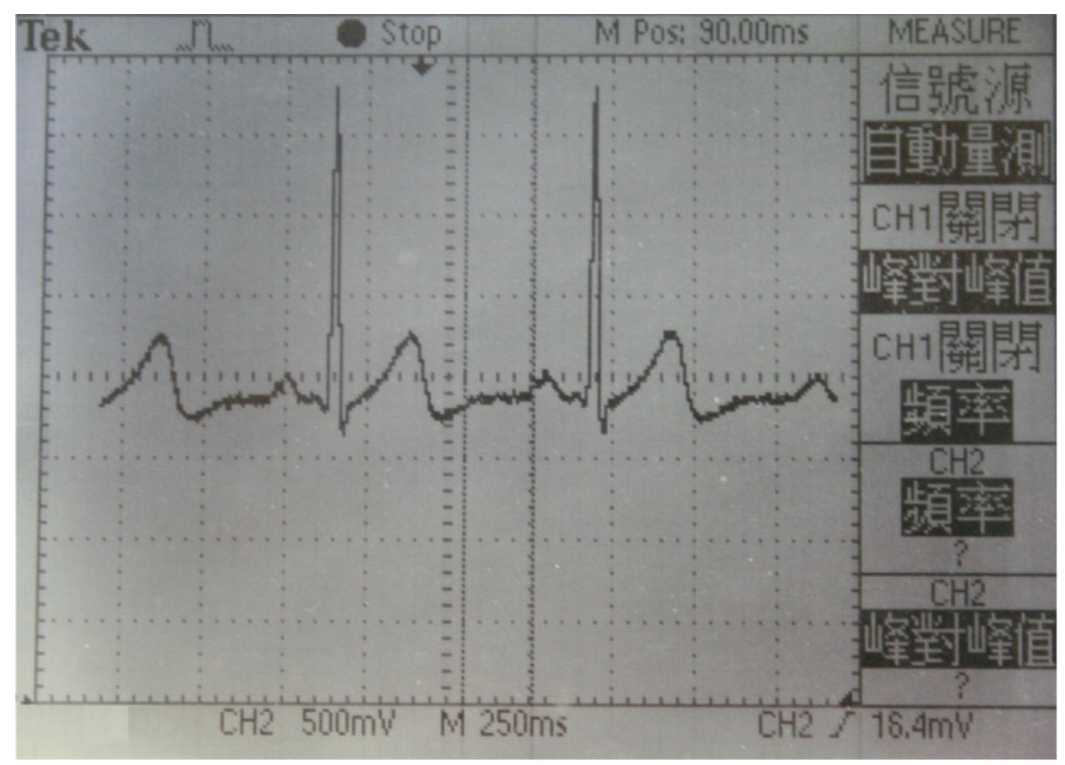

(A)

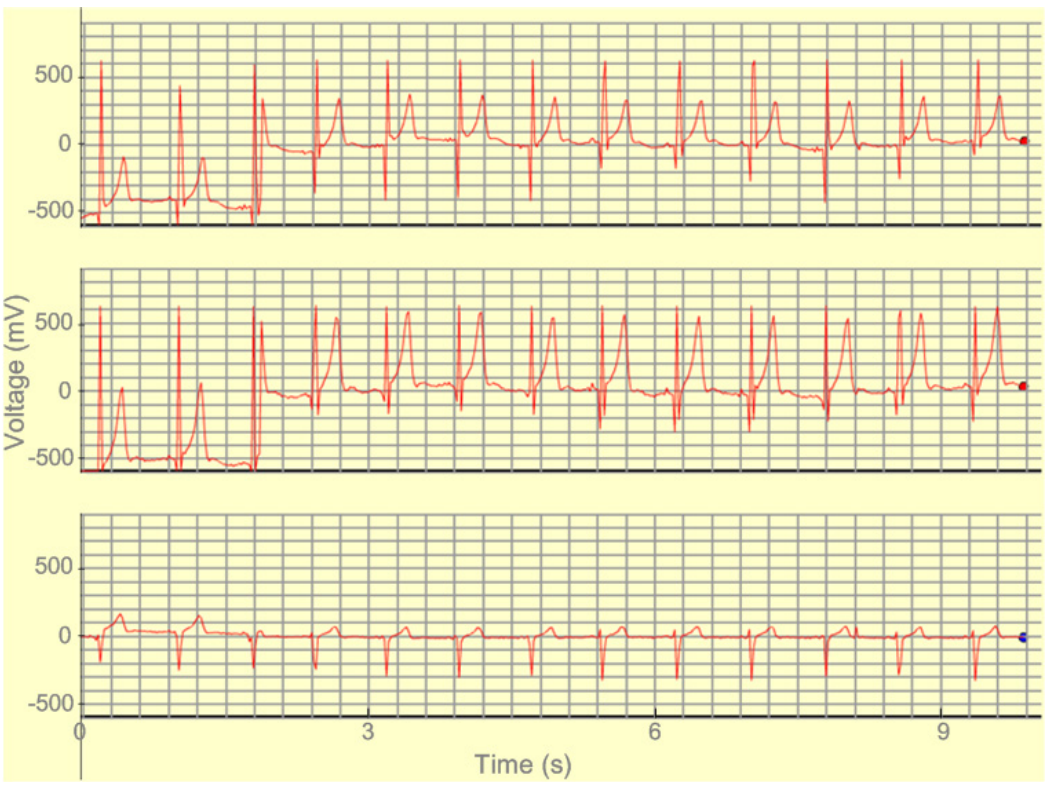

(B)

Fig. 3 The measured ECG signals: (A) A recorded lead II ECG signal is hard copied from the oscilloscope; (B) ECG signals from lead I, lead II, and lead III were recorded simultaneously through wireless transmission. 


\section{DISCUSSION}

A miniature signal-condition module for portable ECG recording was designed and tested. It contained optimal-tuning circuitry of amplification and filtration for pre-conditioning of one-channel ECG signal. By utilizing three modules and a multiplexor, 12-lead ECG has been recorded successfully. As a result, the 12-lead ECG recording device can assist in early diagnosis of myocardial infarction and is aimed for the application of remote home health care.

To achieve the requirements of portable recording, all components of the circuit were carefully selected to minimize the size and power consumption. The signal-condition module was implemented on a printed circuit board $(\mathrm{PCB})$ to facilitate its duplication and increase its mechanical reliability. Moreover, the modular approach has increased the flexibility by varying its pass-band and gain for other biomedical signals. Therefore, the proposed signal-condition module can be accommodated for various biomedical applications before wireless transmission and provides a general solution for portable applications.

\section{ACKNOWLEDGMENT}

This work was supported by grant 95-2218-E-002-071 from the National Science Council, Taiwan.

\section{REFERENCES}

1. Lai D, Fang Z, Real-time remote monitoring of outof-hospital patients especially with high risk heart diseases, Complex Med Eng 332-335, 2007.

2. deAzevedo DFG, deMoura EP, DeCastro MCF, DeCastro FCC, daRocha MF, Telemedicine: Remote monitoring of cardiac patients, IEEE Eng Med Biol Soc(EMBS) 2:1390-1393, 2003.
3. Engin M, Yamaner Y, Engin EZ, A biotelemetric system for human ECG measurements, Measurement 38(2):148-153, 2005.

4. Hsu HW, Analysis of Wireless Transmitted SEMG and ECG from Low-back Pain Patients, Institute of Biomedical Engineering NTU, Taipei, Taiwan, 2006.

5. Jang JK, Portable Recording System for Electrogastrogram Application, Institure of Biomedical Engineering, NTU, Taipei, Taiwan, 2007.

6. Chien CN, Jaw FS, Miniature ultra-low-power biopotential amplifier for portable applications, Biomed Eng Appl Basis Commun 17(2):44, 2005.

7. Dobromir PD, Neycheva T, Mudrov N, Bootstrapped two-electrode biosignal amplifier, Med Biol Eng Comput 46(6):613-619, 2008.

8. Spinelli EM, Martinez N, Mayosky MA, Pallas-Areny $\mathrm{R}$, A novel fully differential biopotential amplifier with DC suppression, IEEE Trans Biomed Eng 51(8):14441448, 2004.

9. Nikola J, Ratko P, Strahinja D, Dejan PB, A novel ACamplifier for electrophysiology: Active DC suppression with differential to differential amplifier in the feedbackloop, IEEE Eng Med Biol Soc (EMBS) 4:3328-3331, 2001.

10. Webster JG, Medical Instrumentation, Application and Design, John Wiley and Sons, New York, pp. 241-245, 1998.

11. Heden B, Ohlin H, Rittner R, Edenbrandt L, Acute myocardial infarction detected in the 12-lead ECG by artificial neural networks, Circulation 96(6):1798-1802, 1997.

12. Menown IB, Mackenzie G, Adgey AA, Optimizing the initial 12-lead electrocardiographic diagnosis of acute myocardial infarction, Eur Heart J 21(4):275283,2000

13. Smit HW, Verton K, Grimbergen CA, A low-cost multichannel preamplifier for physiological signals, IEEE Trans Biomed Eng 34(4):307-310, 1987.

14. Cutmore TR, James DA, Identifying and reducing noise in psychophysiological recordings, Int $J$ Psychophysiol 32(2):129-150, 1999. 